\title{
Programs for quicker relationships
}

\author{
Norman I. Platnick
}

Hennig86, 1.5. By J.S. Farris, 41 Admiral Street, Port Jefferson Station, New York 11776. IBM-PC and compatibles. $\$ 50$.

PAUP, 3.0. By D.L. Swofford, 607 East Peabody Drive, Champaign, Illinois 61820. Apple Macintosh, and IBM-PC and compatibles. \$50. Upgrades \$25.

Hypotheses about the phylogenetic relationships of organisms are central to many aspects of evolutionary biology, from systematics and biogeography to studies of speciation and evolutionary rates. Most systematists today group species into higher taxa (genera, families and so on) solely by their degree of phylogenetic relationship. This approach is commonly called cladistics, because its proponents include in classifications only clades groups of species that are all more closely related to each other than any of them are to species excluded from the group.

The evidence for clades consists of characters that are unique to the groups thus constituted, such as mammary glands (unique to mammals) or spinnerets (unique to spiders). In practice, a matrix of taxa and their characters is constructed, and that information is used to infer a branching diagram (cladogram) specifying all the clades supported by the data. In some cases, usually involving relatively small numbers of taxa and characters that are in complete (or nearly complete) agreement, it is possible to construct a cladogram by hand. Indeed, the cladograms produced by the founder of cladistics, the late German entomologist Willi Hennig, were all inferred entirely by hand. Those inferences involve a parsimony procedure: joint possession of a character is taken to be evidence of common ancestry unless other characters disagree with that grouping.

\section{Larger data sets}

For larger data sets, or those in which several characters disagree, discovery of the correct cladogram(s) by hand is impractical. For just nine taxa, for example, there are over two million possible cladograms, even counting only those arrangements that are completely specified (that is, are dichotomous, having but two branches arising from each node). Moreover, the problem of finding the most parsimonious (shortest) cladogram for a given data set has been shown to be NPcomplete, meaning that an efficient algorithm which will always generate the correct solution is unlikely to exist. Given these difficulties, it is hardly surprising that cladists routinely depend on computer programs to analyse their data.

The two new programs reviewed here - the 1.5 edition of Hennig86 and the 3.0 edition of PAUP (Phylogenetic Analysis Using Parsimony) - both run on micro- computers and provide an enormous increase in the accuracy and efficiency with which solutions can be obtained. Each of them implements both exact and approximate algorithms.

The exact algorithms use a branch-andbound strategy to eliminate suites of cladograms that cannot possibly be among the shortest arrangements. Branch-andbound implementations in previous microcomputer programs* have been either too slow for problems involving more than a dozen taxa, or have produced output requiring hours of hand analysis. For example, the PENNY program in $\mathrm{J}$. Felsenstein's PHYLIP package, and an earlier version of $P A U P$, each produce 54 cladograms for a data set involving 12 taxa and 42 characters. But not all of those cladograms are different; although they are treated as completely dichotomous, some of their branches are unsupported by characters (are of zero length), and when those branches are collapsed into polychotomies, identical cladograms often result. Several hours of work are required to determine that of the 54 cladogramıs, only 19 are distinct. The new programs produce just those 19 distinct cladograms, with no redundancy. The older programs could store and output only 100 cladograms, even if many more were discovered, and because of redundancy those 100 were often an insignificant sample of the actual range of equally parsimonious solutions. The new programs can store many more cladograms $(2,500$ or more, depending on the number of taxa), all of which are really different.

The efficiency with which these exact algorithms work is data-dependent. For data sets with little disagreement among the characters (that is, with an internal consistency near 1.0), astonishingly quick results are possible; both $P A U P$ and Hennig86 obtained exact answers for a 21taxon data set with a consistency index of 0.82 in less than 15 seconds. Hennig86 solved a noisy 20-taxon data set (consistency index 0.38) in just four hours almost three times as fast as the best previous program, and with complete solution of 90 different cladograms, rather than partial solution of 100 with duplicates.

The approximate algorithms make initial estimates of the best cladograms and then systematically rearrange their branches in search of improved arrangements. Unlike the branch-and-bound approaches, these algorithms are not guaranteed to produce optimal results, but in practice they do extremely well, and run very quickly; with large data sets they are the only feasible approach. Each program offers many combinations of options for repeating the analyses with different initial estimates of the correct cladogram, providing diligent users with exceptionally good chances of finding optimal solutions. For example, out of 15 data sets that are too large for exact solutions to have been found, Hennig86 and the new PAUP each found, in five cases, results better than had been obtained from any previously available mainframe or microcomputer program.

\section{Greater weight}

Both programs allow users to give greater weight to some characters than to others, and to treat multi-state characters as additive (such that changing from state $A$ to $C$ requires an intermediate change to state B) or non-additive (such that any state can transform directly into any other). I ran a total of 86 analyses on Hennig86, involving all combinations of weighted/unweighted and additive/nonadditive characters, in which the results of four different approximate options were compared to an exact solution. In every case at least one of the approximate options found the shortest cladogram. Moreover, there was no instance in which at least one of the approximate options did not find all of the shortest cladograms.

Both programs are loaded with features that will endear them to users. PAUP includes intuitive pull-down menus, and a built-in editor with syntax error pointing; the Macintosh version will interface directly with the forthcoming update of the excellent MacClade program, which allows interactive cladogram rearrangement and character-change tracing on the Apple machines. Hennig86 includes a smooth implementation of successive approximations weighting, allowing users to determine which of the equally parsimonious cladograms are supported by the least noisy characters, and has its own MacClade-like facility for interactive tree rearrangement and character tracing.

As for efficiency, Hennig86 on an 80386-based PC analysed a suite of 25 data sets over three times faster than the older PHYSYS program on a CYBER mainframe, even though it was saving ten times as many cladograms and finding better solutions for six data sets. The moral dump the mainframe, get a fast PC and enjoy using these programs.

*Earlier programs were discussed by M. Luckow and R.A. Pimentel in Cladistics 1, 47-66 (1985); W.L. Fink in Science 234, 1135-1139 (1986); and myself in Cladistics 3, 121-144 (1987).

Norman I. Platnick is Chairman of the Department of Entomology at the American Museum of Natural History, New York, New York 10024, USA. 